\title{
Accessing the particular from the general: The power of distinctiveness in the context of organization
}

\author{
R. REED HUNT and REBEKAH E. SMITH \\ University of North Carolina, Greensboro, North Carolina
}

\begin{abstract}
Recall is inversely related to the number of items sharing a cue. The limiting case of unique cuetarget relationships supports extremely high levels of recall, particularly when the cue is self-generated. This fact is incongruous with the importance assigned to the construct of organization in memory theory. Further, self-generated unique cue-target relationships tend to be idiosyncratic, implying that the power of unique cues should be limited to cases of self-cued memory. The experiments presented here suggest a role for organization that reconciles the fact of unique cue effectiveness with the importance of organization to memory. Two new findings are reported: Unique cue production enhances target encoding; and general cues can access particular encodings. The data are further tribute to the importance of simultaneous organizational and distinctive processing and recommend a new perspective on the function of organization in memory.
\end{abstract}

Cue effectiveness in memory is a function of the uniqueness of the cue-target relationship. For example, we have known for some time that the power of a category label as a cue is inversely related to the number of category instances presented at study (Tulving \& Pearlstone, 1966; Watkins \& Watkins, 1975). Furthermore, Moscovitch and Craik (1976) explicitly manipulated the number of targets paired with a cue and found that unique cues produced better memory than shared cues. Stein (1977) induced unique cue-target relationships at encoding by requiring subjects to rate the goodness of similes (e.g., "A bumper is like a statue."), and then tested recall of the second word (statue) with the first word (bumper) as a cue. Performance was much better under these conditions than when the same pair of words was rated for the similarity of hardness at encoding, presumably because the simile established a unique relationship between the cue and target.

Perhaps the most impressive demonstration of the effectiveness of unique cues is the research of Mäntylä (1986; Mäntylä \& Nilsson, 1983, 1988). Mäntylä’s paradigm required the subjects to generate known attributes of the target words at study. These attributes then were returned to the subjects to be used as cues for recall of the study words. Unprecedented levels of recall occurred in the presence of these self-generated cues, ranging as high as $90 \%$ correct for 600 unrelated words (Mäntylä, 1988). Mäntylä and Nilsson $(1983,1988)$ provided evidence

This research was supported by a grant from NICHHD (No. HD25587). G. Craik, R. Guttentag, T. Mäntylä, D. Nelson, and $D$. Rubin provided constructive criticism of a previous version of the manuscript. Correspondence should be addressed to R. R. Hunt, Department of Psychology, University of North Carolina, Greensboro, NC 27412 (e-mail: huntrr@iris.uncg.edu). that cue-target uniqueness was the principal contributor to this effect.

The self-generated cue paradigm is important beyond its contribution to the question of uniqueness in that it opens a window on an important source of cues inherent to the encoding process. Perception and comprehension of an item, which we take to be encoding, entail production of information constituting the meaning of the item. This information not only represents encoding but also is a potential source of cues. When these cues are unique, as in Mäntylä's paradigm, they seem to produce near-perfect performance.

In spite of the intuitive plausibility of uniqueness as a component of cue effectiveness, several puzzles arise when this fact is considered in the broader context of memory theory and research. The first puzzle stems from the fact that the most effective self-generated cues in Mäntylä's paradigm were unique in the sense of being idiosyncratic; that is, the overlap of cues across subjects was relatively small (Mäntylä \& Nilsson, 1983, 1988). On the principle of encoding specificity, one then expects these unique cues to be effective only in the case of selfcued memory.

Is the power of unique, self-generated cues unavailable to someone else trying to cue your memory? This question is relevant not only for laboratory methodology, but also, and perhaps more importantly, for the analysis of memory as applied to social interaction. In social interaction, other people do not have access to your unique cues, and indeed, many, if not most, interactions begin with more general cues (e.g., "What happened at the meeting on Friday?"). These general cues presumably are more consensual in that you and I will share the encoding of an episode ("the meeting on Friday"), but what role does the unique cue encoding play? 
The relationship between unique and more general cues leads us to the second puzzle, the juxtaposition of the effectiveness of unique processing against the presumed importance of organizational processing. Organization is among the sacrosanct constructs in memory theory, and justifiably so given the voluminous evidence that variables affecting organization enhance memory. However, the function of the construct of organization is to account for the encoding of similarities among items. Unique cues emphasize just the opposite in that the dominant sense of uniqueness in the literature is that the cue-target relationship differs for each pair of items. If this unique relationship is critical for cue effectiveness, then what is the basis of the positive contribution from organization?

The dilemma posed by the second question is a manifestation of the broader tension between the constructs of organization and distinctiveness, and we shall approach the issue from the general perspective that both types of processes contribute to memory of a given item (Hunt \& McDaniel, 1993). Implementation of this approach begins with the assumption that self-generation of cues affects encoding of the target item in addition to establishing the cue-target relationship. Production of a unique cue induces distinctive encoding of the target in that the attributes of the encoding represented by the distinctive cue are not shared by other target items. Production of a shared cue, however, encourages organizational processing in that the attributes of targets representing the shared cue are similar or identical. The shared cue condition will be at a relative disadvantage because of the impoverished encoding of distinctive information that serves a discriminative function in retrieval (Hunt \& McDaniel, 1993). If, as speculated here, cue production influences target encoding as well as the cue-target relationship, we would expect production of a distinctive cue to yield better free recall than production of a shared cue.

Confirmation of this prediction would essentially replicate previous demonstrations that distinctive processing of categorized words yields better memory than organizational processing (Einstein \& Hunt, 1980; Hunt \& Einstein, 1981). This result is evidence for the benefit of combined distinctive and organizational processing in that the categorized list encourages organizational processing while the unique cue production encourages distinctive processing. Nonetheless, the question remains: What is the function of organization?

Suppose the organizational cue were capable of reintegrating the earlier distinctive processing? That is, what if the general cue were capable of accessing the particular encoding? If so, answers to both of the aforementioned puzzles are available. Part of the function of organization would be to provide the context for distinctiveness (Craik \& Jacoby, 1979). Distinctive processing is the processing of differences aligned to some context (Medin, Goldstone, \& Genter, 1993), and that context could be the dimension of similarity underlying organization. Reinstatement of the organizational cue restores that con- text and the distinctive processing. In addition, the organizational processing is likely to be more consensual across people than is distinctive processing, so that someone else's organizational cue is likely to be identical to your own. If this general cue is capable of accessing the particular encoding, the power of idiosyncratic distinctive processing could be reinstated by someone else's shared cue, thereby increasing the generality of distinctive processing in the use of memory.

The foregoing speculation leads to several predictions. First, the consensuality of self-produced shared cues should be greater than the consensuality of unique cues. It then is trivial that someone else's shared cue will be as effective as your own, but less obvious is whether the shared cues will produce the same level of recall as the unique cues when both types of processing occurred at encoding. The following experiments will demonstrate that this in fact is the case.

The first experiment will show that self-generated unique cues are more effective than self-generated shared cues and that the effectiveness of the shared cues does not differ as a function of self- versus other-generation. Surprisingly, however, the unique cues generated by someone else were quite effective even though these cues were not the same as those generated by the subject. The second experiment will show that this latter finding was due to the availability of shared cues in the first experiment. Finally, the third experiment will compare directly the effectiveness of shared and unique cues following the production of both. When the subject has generated both a shared cue and a unique cue, the two are equally effective, suggesting that unique cue production at encoding confers benefits that can be realized through a shared cue at test

\section{EXPERIMENT 1}

In the first experiment, subjects produced either shared or unique cues to categorized word lists at study. Unique cues were defined as attributes of a category instance not shared by any of the other category members in the list, whereas shared cues were defined as attributes shared by all presented members of the category. Recall was cued by the subjects' own cues, by someone else's cues, or by free recall instructions. The hypotheses of interest were, first, that self-produced shared cues would produce poorer performance than would self-produced unique cues, replicating prior research (e.g., Moscovitch \& Craik, 1976). Second, unique cue production at study would lead to better free recall than would shared cue production, implicating encoding processes in the unique cue effect. Third, the agreement among subjects on shared cues would be greater than on unique cues, and consequently, recall cued by someone else's shared cue would exceed recall cued by someone else's unique cue. Finally, and most speculatively, shared cues would reinstate the processes of unique encoding. This last hypothesis predicts that someone else's shared cue would be much 
more effective if the study task required unique rather than shared cue production.

\section{Method}

Subjects and Design. The subjects were 96 volunteers from an introductory psychology class who were assigned in equal numbers to eight conditions. The conditions were defined by the orthogonal combination of study task and cue type. The study tasks were to produce either a unique or a shared cue to the target words. At test, subjects received one of four types of cues: self cues (unique or shared, depending on the study task), unique other, shared other, or free recall instructions. In the case of other cues, where the subject received cues produced by someone else, each subject was yoked to a self-cue subject. Both study task and cue type were between-subjects manipulations.

Materials and Procedure. The study lists were composed of instances of intermediate frequency (4-16) from the Battig and Montague (1969) category norms. The lists contained 50 items, 5 from each of 10 different categories, and two different lists were counterbalanced across subjects. The lists were presented in blocked fashion with the five words from a given category appearing on each page of a booklet.

Subjects in the unique study task condition were instructed to write one thing they knew about each of five words on a page that was not true of any of the other words on the page. Shared study task instructions were to write one thing common to all five words on each page of the booklet. The instructions did not mention the subsequent memory test. The cue generation phase was subjectpaced. Following a 5-min retention interval, during which credit forms were completed, the recall test was given. Subjects receiving cues were given a booklet containing the appropriate cues, either their own or someone else's. The cues were blocked at test just as at study, but the order of categories was different in study and test. Free recall subjects were asked to write all words they could remember.

\section{Results}

The mean proportion of correct cued recall is presented in Table 1 as a function of study task and cue type. The main effect of study task was reliable ( $F=176.99$, $M S_{\mathrm{e}}=.05$ ), indicating better recall following the unique study than shared study. Cue type also exerted a reliable effect on performance $\left(F=31.95, M S_{\mathrm{e}}=.05\right)$, but the interaction between study task and cue type was not reliable.

The specific predictions motivating the experiment were tested by planned comparisons in the form of Newman-Keuls tests. These analyses revealed that self unique cues led to reliably higher recall than self shared cues, replicating previous work on unique cues. The same results occurred in free recall. This new finding indicates

\section{Table 1}

Proportion of Correct Recall as a Function of Study Task and Cue Type in Experiment 1

\begin{tabular}{lcc} 
& \multicolumn{2}{c}{ Study Task } \\
\cline { 2 - 3 } \multicolumn{1}{c}{ Cue Type } & Unique & Shared \\
\hline Self & $.97( \pm .02)$ & $.59( \pm .07)$ \\
Unique other & $.85( \pm .03)$ & $.51( \pm .08)$ \\
Shared other & $.86( \pm .03)$ & $.58( \pm .07)$ \\
Free recall & $.60( \pm .09)$ & $.35( \pm .07)$ \\
\hline
\end{tabular}

Note-Numbers in parentheses are confidence intervals.
Table 2

Correct Recall Conditionalized on Cue Consensus in Experiment 1

\begin{tabular}{lcc}
\hline & \multicolumn{2}{c}{ Cue Type } \\
\cline { 2 - 3 } Cue Consensus & Unique & Shared \\
\hline Consensual & $.91( \pm .02)$ & $.63( \pm .05)$ \\
Nonconsensual & $.85( \pm .03)$ & $.35( \pm .06)$ \\
\hline
\end{tabular}

Note-Numbers in parentheses are confidence intervals.

that the benefits of unique cue production can be present in the absence of the explicit cues at test and may reflect the influence of unique and shared cue production on encoding.

The more speculative hypothesis about recall was that shared cues could be used to reinstate unique encoding. Of interest here is the comparison between the groups that received other shared cues following unique study and the groups that received other shared cues following shared study. As is evident in Table 1, other shared cues were reliably more effective following unique study than following shared study.

One result depicted in Table 1 was completely unanticipated and potentially problematic. Performance with other unique cues following unique study was unexpectedly high and did not differ reliably from recall to other shared cues following unique study. We assumed that unique cues would be relatively idiosyncratic, rendering them of little use to someone else. Examination of the data on cue consensus becomes important if we are to see whether we were wrong in making that assumption.

Cue consensus was computed for subjects receiving someone else's cues by determining the overlap between the cues produced by the 2 subjects at study. Consensus could be computed only within the same study task. That is, the data are drawn from subjects receiving other unique cues at test following unique cue production at study and from subjects receiving other shared cues at test following shared cue production at study. In scoring these data, we counted slight transformations as identical. The most frequent instances of these transformations were singular versus plural, noun versus adjective, and variations in verb tense. Almost all shared cues essentially were labels for the categories, whereas the unique cues were quite idiosyncratic. The overlap for unique cues was only .12, whereas the overlap for shared cues was .77. These results confirm our original hypothesis that shared cues would enjoy greater consensus across subjects than would unique cues, but at the same time deepen the mystery of the effectiveness of someone else's unique cues.

The puzzle is highlighted by an analysis of recall cued by someone else's cue conditionalized on cue consensus. These data are shown in Table 2 as a function of study task and cue consensus. Not only were study task ( $F=$ 98.77, $\left.M S_{\mathrm{e}}=.02\right)$ and consensus $\left(F=16.69, M S_{\mathrm{e}}=\right.$ $.02)$ reliable, but so too was the interaction between them $\left(F=6.87, M S_{\mathrm{e}}=.02\right)$. Newman-Keuls analysis of the 
data indicated a reliable difference between consensual and disparate shared cues but no reliable difference for unique cues. Performance with the unique cues following unique cue production was very good regardless of whether the cue was one the subject actually produced.

\section{Discussion}

The results of the first experiment replicate and extend previous research on unique cues. Self-produced unique cues yielded considerably higher recall than did self-produced shared cues. Given that the unique cues were generated to a categorized list, this result can be seen as another instance of the combined effects of organization and distinctiveness exceeding the effects of organization alone (Hunt \& Einstein, 1981). Furthermore, the fact that the superiority of unique cue production carried over to free recall indicates that processing of unique cue-target relationships at study influences encoding of the target item. If this is the case, the question of accessing unique encoding with the shared cue becomes important, particularly given the differential consensuality of unique and shared cues. That is, production of a unique cue enhances the potential for subsequent memory relative to production of a shared cue, but realization of this potential would be more general if the unique encoding could be accessed by not only the unique cue, but also the more consensual shared cue. In short, someone else would have a greater chance of cuing your unique encoding.

The data strongly support this possibility. Recall following unique cue production given someone else's shared cue was very good and far exceeded recall following shared cue production. We assume that category information was spontaneously encoded in the unique cue conditions given the categorized lists (Hunt \& Seta, 1984) and that the consensuality of this category information across subjects means that the shared cues for different subjects were essentially identical. Thus, the unique cue study condition allows encoding of shared cue information that corresponds to the information of the shared cue production study condition. The results then clearly indicate that the shared cue can access the unique encoding.

One aspect of the data was completely unexpected. Subjects given someone else's unique cue following unique encoding performed at an extremely high level. This result is surprising in light of the assumptions underlying the otherwise successful predictions. The relatively low levels of consensus on unique cues should have produced low levels of recall. The second experiment was designed to diagnose the effectiveness of someone else's unique cue.

\section{EXPERIMENT 2}

Consideration of the results of the unique other cues from the first experiment combined with the evidence that shared cues could access the unique encoding lead to the strong suspicion that the test procedure allowed subjects in the unique condition to ascertain the category represented by the other cues. The test cues in Experiment 1 were blocked just as the study items were blocked. The subjects could make use of this information at test by using the other cues to discern which category was being cued, and then use the category information for recall. This hypothesis is important not only as an explanation of the data from Experiment 1, but also because it suggests that the category cue following unique encoding may be as effective as the unique cue.

An alternative, and more sinister, hypothesis is that performance with unique other cues in Experiment 1 represents associative guessing. The sinister aspect of this hypothesis is that it would also apply to the self cue data. Consequently, discounting the associative guessing hypothesis is important lest all effects of self-generated cues be interpretable without appeal to episodic memory.

If our hypothesis about the blocking of cues is valid, then presenting cues individually in a random order should reduce the effectiveness of the other unique cues. The category of the target should be less obvious in the presence of one cue than it was with five cues. Alternatively, if single cues were used to guess the item, then individual presentation of the items should not change the results.

\section{Method}

Subjects and Design. The subjects were 60 volunteers from introductory psychology who were randomly assigned to one of six conditions. The conditions were defined by an orthogonal combination of cue type (self, other, free recall) and cue presentation (blocked, discrete). Two free recall groups were included simply to balance the design; these two groups were treated identically.

Materials and Procedure. At study, all subjects received the same words, blocked by category, and the unique study task instructions used in Experiment 1. Subjects who subsequently received blocked cues at test wrote their study task responses in a booklet just as in Experiment 1. Subjects who were to receive discrete cues at test wrote each of their study task responses on a separate card. The study task was followed by a 5 -min interval during which subjects completed credit forms, and then a surprise recall test was administered.

At test, subjects in the blocked cue presentation condition received a booklet of 10 pages, each page containing five cue words just as in Experiment 1. The discrete cue presentation subjects were given a deck of 50 cards with one cue written on each card. The discrete cues were presented randomly with the restriction that no more than two cues from a category could appear contiguously. Self cue subjects received their own cues and other cue subjects were yoked to a self cue subject as in Experiment 1. Subjects were instructed to write words from the study list of which they were reminded by the cues. The responses were written on the booklet or card, and in neither blocked nor discrete conditions were subjects allowed to go back to a page or card once they had responded. The free recall subjects wrote their responses on a sheet of paper, and again, two separate free recall groups were run in order to balance the design.

\section{Results}

The mean proportion of correct recall is presented in Table 3 as a function of cue type and cue presentation. Both cue type $\left[F(2,54)=15.21, M S_{\mathrm{e}}=.02\right]$ and cue presentation $\left[F(1,54)=29.97, M S_{\mathrm{e}}=.02\right]$ were reliable. More 
Table 3

Correct Recall as a Function of Cue Type and Cue Presentation in Experiment 2

\begin{tabular}{lcc}
\hline & \multicolumn{2}{c}{ Cue Presentation } \\
\cline { 2 - 3 } Cue Type & Blocked & Discrete \\
\hline Self & $.93( \pm .02)$ & $.88( \pm .02)$ \\
Other & $.87( \pm .02)$ & $.46( \pm .06)$ \\
Free recall & $.58( \pm .07)$ & $.56( \pm .06)$ \\
\hline
\end{tabular}

Note-Numbers in parentheses are confidence intervals.

important for the purposes of this experiment was the reliable interaction between the two variables $[F(2,54)=$ $\left.17.45, M S_{\mathrm{e}}=.02\right]$. Subsequent Newman-Keuls analysis within blocked cue presentation showed no reliable difference between self and other cue types, but both self cues and other cues were reliably better than free recall. The discrete cue presentation yielded a different pattern. Self cues reliably exceeded performance with other cues and free recall, and the difference between free recall and other cues was not reliable. Thus, discrete cue presentation rendered the other cues ineffective relative to self cues.

A similar conclusion emerged from the analysis of cue consensus and recall. The mean overlap for discrete and blocked other cue conditions, .11 and .14, respectively, did not differ. There would be no reason to have expected any difference in cue overlap since the discrete and blocked presentation groups were treated identically at study. Correct recall conditionalized on cue consensus is presented in Table 4 as a function of cue presentation and cue consensus. The main effects of cue consensus $\left[F(1,18)=13.23, M S_{\mathrm{e}}=.07\right]$ and cue presentation $\left[F(1,18)=16.73, M S_{\mathrm{e}}=.10\right]$ were reliable, but the reliable interaction between the two variables $[F(1,18)=$ 14.67, $\left.M S_{\mathrm{e}}=.07\right]$ is more informative. Newman-Keuls tests yielded no reliable difference between consensual and nonconsensual cues for blocked cue presentation, but consensual cues produced reliably higher recall than did nonconsensual cues with discrete cue presentation.

\section{Discussion}

The effectiveness of unique other cues following unique study clearly depended on blocked presentation of the cues. Discrete cue presentation reduced other cue performance substantially, whereas blocked cue presentation replicated the relatively high level of recall found in Experiment 1. As in Experiment 1, the relatively high level of other cue performance occurred in spite of minimal overlap between the cues produced by the subject and the cues the subject received. Furthermore, cues that were identical to those generated by the subject were not reliably more effective than cues the subject had not produced in the blocked cue presentation condition. We must find something other than the explicitly provided cue that would support the performance with blocked cues.

The most reasonable candidate is category information. We know from the literature on organization that subjects spontaneously encode category information (e.g.,
Hunt \& Seta, 1984), and although the subjects probably could not remember all 10 of the categories spontaneously at test, a block of five different cues could be used to determine the particular category in question. At that point, the unique other cues could be used with the category information to converge on a target even when the unique other cue was not one produced by the subject.

Consistent with this interpretation, the discrete presentation of other cues substantially reduced recall in the other cue condition. Furthermore, with discrete presentation, the effectiveness of a cue depended heavily on that cue being one produced by the subject. Our hypothesis was that discrete presentation would reduce the subjects' ability to discern the category; thus, removing the category information would reduce recall. Note that if the other cue performance reflected associative guessing to the cue, there would be little reason to expect a decline in performance with discrete presentation, and therefore the data from Experiment 2 do nof support such a hypothesis.

On our interpretation, the data of Experiment 2, as well as those from the unique other cue condition of Experiment 1 , are further examples of general cues effectively accessing specific encodings. Unique other cues, when presented in blocked fashion, provide enough information to access the general categorical cue, which then provides access to the specific target encoding. We suggest an analogy to the social situation in which someone provides his/her own distinctive cue with little explicit context—such as in a friend's remark, "The gumbo was far too mild." By itself, this remark cues no specific episode, but the remark in conjunction with other information, perhaps the person making the remark and where the two of you last shared gumbo, accesses "Paul's dinner party." At this point, you may access your encoding of the gumbo as being just right. Of course, the social situation allows a more direct response to idiosyncratic, unique cues, such as, "What are you talking about?" but the point is that retrieval of specific encoding by general cues is an important phenomenon.

\section{EXPERIMENT 3}

The purpose of the final experiment was to contrast directly the effects of unique and shared cues under circumstances in which each subject explicitly engaged in both kinds of processing at study. If, as we have speculated, a shared cue can reintegrate distinctive processing, the advantage of self unique over self shared cues should disappear when both types of cues were produced at study.

Table 4

Correct Recall Conditionalized on Cue Consensus as a Function of Cue Presentation in Experiment 2

\begin{tabular}{lcc}
\hline \multirow{2}{*}{$\begin{array}{c}\text { Cue } \\
\text { Consensus }\end{array}$} & \multicolumn{2}{c}{ Cue Presentation } \\
\cline { 2 - 3 } Consensual & Blocked & $\frac{\text { Discrete }}{.90( \pm .02)}$ \\
Nonconsensual & $.87( \pm .03)$ & $.84( \pm .03)$ \\
\hline
\end{tabular}

Note-Numbers in parentheses are confidence intervals. 
Requiring the subject to produce both a unique and a shared cue to each target at study assures us that both organizational and distinctive processing occurred at encoding. Thus, we can make a direct comparison of the cue effects at test unconfounded by possible differences in encoding processes.

Another change in procedure from Experiments 1 and 2 allowed the number of generated cues and items recalled per cue to be equated in the unique and shared conditions. At study, one of the items was spatially separated from the other four items in the category. The unique and shared cues were generated to this item relative to the other four, and the single item was recalled to the cue. We have used this manipulation in other unpublished experiments to assure that the advantage of unique over shared cues was not due to the number of targets per cue. In those experiments, the unique cue advantage was as great as that in Experiments 1 and 2, even though only one target was recalled to each shared cue.

\section{Method}

Subjects and Design. The subjects were 50 volunteers from introductory psychology. All subjects performed both the shared and unique study tasks on each target item. The experimental conditions were defined by the cue type received at test, and these conditions were entirely between subjects. Thus, the design was a one-way factorial with five levels of cue type: unique self, unique other, shared self, shared other, and free recall.

Materials and Procedure. Five instances of 20 categories comprised the 100 word lists. The categories were presented in block fashion so that one of the words, the target word, was spatially separated from the other four instances of the category. The target word was four spaces directly above the other four words, which were separated from each other by a single space. Each of the five words in the category served equally often as the target word across subjects. In the unique study task, subjects were instructed to write one thing they knew about the separated word that was not something they knew about the other four words. The shared study instructions were to write one thing about the separated word that was also true of the other four items.

The experiment was run on an IBM XT computer controlled by the Micro Experimental Laboratory program. At study, the five words from each category appeared on the monitor until the subjects typed their response. Each subject saw the list twice, once to perform the shared study task and once to perform the unique study task. The order of study tasks was counterbalanced across subjects, and the order of category presentation within the list was random for each list presentation.

Following the study phase, the appropriate cues were presented one at a time in a random order. Subjects typed their responses to the cues. Subjects who received cued recall tests first responded to the 20 cues with the single target item and then were given a second recall test for the four nontarget items from each category. The original cues were represented for the nontarget test. Subjects given free recall instructions first typed their target responses and then, with the monitor cleared, typed their nontarget responses. Subjects were run individually.

\section{Results}

Data were analyzed on three dependent measures: target recall, nontarget item recall, and cue consensus for those subjects receiving other people's cues. Each of these analyses will be reported separately.

Target recall. The average proportion of targets correctly recalled is presented in the top portion of Table 5 . Cue type exerted a reliable effect on recall $[F(4,45)=$ $\left.32.74, M S_{\mathrm{e}}=.01\right]$. Pairwise comparisons of the means yielded no reliable difference between self-produced shared and unique cues. Other people's shared cues also did not differ reliably from these two conditions. Other people's unique cues produced reliably poorer recall, and this performance did not differ reliably from free recall.

Nontarget item recall. The bottom row of Table 5 depicts performance on the four nontarget items as a function of cue condition. Recall of these other items was affected reliably by cue type $\left[F(4,45)=7.74, M S_{\mathrm{e}}=.02\right]$, and as can be seen in Table 5, self-produced shared and unique cues produced comparable performance on nontarget items. This conclusion was confirmed by pairwise comparisons. These comparisons also showed no reliable difference between free recall and other people's unique cues.

Cue consensus. The overlap of cues produced and received by subjects who were given someone else's cues was computed in the same way as in Experiments 1 and 2 . The proportion of overlapping cues for subjects receiving someone else's shared cue was .68, and for subjects receiving someone else's unique cues, the overlap was .16. As in Experiment 1, the consensus on shared cues was reliably higher than that on unique cues.

\section{Discussion}

The results of Experiment 3 are a direct demonstration that shared cues can support the same high level of recall as unique cues. The critical factor appears to be itemspecific encoding combined with encoding of the dimension represented by the shared cue. Under these circumstances, self-produced shared cues produced performance comparable to self-produced unique cues. Indeed, even someone else's shared cue led to performance that was not reliably different from that with self-produced unique cues. This result is easily understood in the con-

Table 5

Recall of Targets and Nontargets as a Function of Cue Types in Experiment 3

\begin{tabular}{lcccccc}
\hline & \multicolumn{5}{c}{ Cue Type } \\
\cline { 2 - 6 } & Self Unique & Other Unique & Self Shared & Other Shared & Free Recall \\
\hline Target & $.90( \pm .02)$ & $.55( \pm .07)$ & $.93( \pm .02)$ & $.85( \pm .03)$ & $.47( \pm .08)$ \\
Nontarget & $.45( \pm .06)$ & $.26( \pm .09)$ & $.49( \pm .08)$ & $.43( \pm .06)$ & $.18( \pm .05)$ \\
\hline
\end{tabular}

Note Numbers in parentheses are confidence intervals. 
text of the cue-overlap data, which demonstrated considerable consensus among subjects on shared cues and much less consensus on unique cues. These consensus data, which replicate the findings in Experiment 1, indicate that people are much more likely to agree on the relational encoding of a set of familiar events than on the distinctive attributes of the same events, and in accord with encoding specificity, someone else's relational cue will be more effective than someone else's distinctive cue. If distinctive attributes of the original event were encoded along with the relational property, the relational cue then would support very high levels of performance.

The analysis of recall for items other than the target indicates that the distinctive processing is specific to the target item. Even though the unique task is similar to the shared task in requiring a judgment of the target relative to the other four items, the benefit of the distinctive processing accrues only to the target item. Processing of the nontarget items in the unique study task probably is dominated by organizational processing in that detection of differences between the target and the four other items requires processing of the shared attributes among these four items. These data thus are consistent with the view that distinctive and organizational processing are qualitatively different.

\section{GENERAL DISCUSSION}

Unique cue-target relationships produce much better recall than cues that are shared by several targets. The most impressive demonstration of this fact comes from Mäntylä's (e.g., 1986) self-produced cue paradigm, which we adapted for use in our experiments. Our results were consistent with previous research in showing a substantial advantage for the self-produced unique cue over the self-produced shared cue. Indeed, the very high levels of recall, which replicate Mäntylä's work, reflect the power of normal adult memory not usually seen in laboratory settings and recommend the paradigm as a valid model of perception/comprehension followed by memory. But the fact of unique cue effectiveness is a bit curious when considered in the broader context of both the pragmatic issue of the social use of memory in which someone else provides the cues and in the theoretical context of organization. Self-produced unique cues led to nearly perfect recall whereas self-produced shared cues yielded lower levels of performance. Yet, the unique cues were considerably more idiosyncratic than the shared cues, suggesting that the unique cues would be of use only with self-cued recall. Further, the shared cue corresponds to organizational processing, a condition traditionally assumed to be favorable for memory.

Two new facts from our experiments reconcile these curiosities with previously known facts. First, the production of unique cues at study enhances free recall, indicating that the encoding of the target item is influenced by production of unique cues. This effect is not surprising in light of either levels of processing (Craik \& Lockhart, 1972) or research on distinctive and organizational processing (Hunt \& Einstein, 1981), but it is important to realize that the effect of unique cues is not just on the cue-target relationship but also on the target encoding. Craik (1979) suggested that encoding variables establish a potential for recall the realization of which depends on the cuing conditions. In our case, one might say that encoding both distinctive and organizational attributes of an item establishes a greater potential for recall than encoding just the organizational attributes.

The second new fact in our data now becomes extremely important. The distinctive encoding engendered by unique cue production can be accessed by the more general cue. Direct evidence to this effect was provided in Experiments 1 and 3, and indirect evidence comes in the form of our interpretation of the unique other cue data from Experiment 1, an interpretation supported by the data of Experiment 2. Combined with the discovery that unique production enhances target encoding, the fact that general cues access the unique encoding resolves the two puzzles mentioned earlier. Even though my unique encoding of an event may be different than yours, we are likely to share encoding of the more general attributes of a familiar situation. Subsequently, these shared general attributes can reinstate the more precise but more idiosyncratic unique encodings. This view offers a different perspective on the function of organization in memory to which we shall return after discussing potential problems with our interpretation.

\section{Possible Confoundings}

Prior to discussion of more general implications of these data, we shall address two possible confoundings that could compromise our interpretation. The first is the possibility that production of unique cues requires more time than does production of shared cues and that this difference in study time explains the differential cue effectiveness. We have evidence to the contrary from two experiments that were not presented here. In fact, generation of unique cues did require more time than did that of shared cues, but a subsequent analysis of the recall data using study time as a covariate still yielded a reliable superiority for the unique cue. In another study, subjects produced either one or three cues to each target at study. Producing three shared cues required reliably more time than did producing a single unique cue, but recall was reliably better following unique cue production. Thus, although it is true that producing a unique cue required more time than did producing a shared cue, the quantitative difference in study time does not appear to be as crucial as the qualitative difference in processing.

The second issue is the possibility that unique cue effects are mediated by associative guessing, not episodic memory. Essentially, the argument would be that the unique cues are strong associates of the targets and will evoke the targets with a high probability when presented at test. Several reasons exist to question the associative guessing hypothesis. First, the cue generation paradigm asks the subject to produce a response to a target but then to use the response to produce the target at test. If asso- 
ciative guessing were the only mechanism involved, one would have to assume symmetrical associative strength across large numbers of word pairs and people. Such an assumption would be incredible.

Further, Mäntylä and Nilsson (1988) reminded us that the distinction between memory and associative guessing is that associative guessing is context free (hence the name "free association"). Mäntylä and Nilsson then demonstrated a forgetting function over retention intervals for distinctive cues, and on the assumption that different retention intervals reflect contextual drift, argued that an associative guessing hypothesis is wrong.

Moreover, Kelly (1993) discovered that self-generated unique cues produced no priming in a test of free association. Her experiment required generation of unique cues, and following a 2-day delay, the unique cues were returned for either recall or free association. Recall was approximately .50 , whereas only .06 of the old items were produced in free association to the unique cue, a level that did not differ from baseline. In general, then, the contribution of associative guessing to performance does not account for the effectiveness of unique cues.

Finally, the associative guessing hypothesis, when applied to our data, requires that the unique cues be more likely to elicit the target than the shared cues. However, the shared cues were just as effective in Experiment 3 as the unique cues. If the advantage of unique cues over shared cues in other experiments were due to contextfree elicitation of the target by the cue, one would expect that advantage in all circumstances. To the contrary, what mattered in our experiments seems to have been circumstances propitious to the combination of organizational and distinctive processing.

\section{The Function of Organization and Distinctiveness}

Distinctive processing has been defined as the processing of nonoverlapping attributes of elements (Eysenck, 1979; Lockhart, Craik, \& Jacoby, 1976). The encoding of such attributes provides a unique specification of the target element and thus facilitates memory by rendering the target element highly discriminable. If a component of the distinctive processing is available at test as a cue, memory will be enhanced even further as the distinctive cue reinstates the original processing. Such an account of distinctive processing is standard fare (see, e.g., Craik \& Jacoby, 1979; Hunt \& Einstein, 1981), but this description leaves out a critical fact about distinctiveness. Distinctiveness is always relative to a particular context (Craik \& Jacoby, 1979).

Consider, for example, the self-generated cue of red to the target word salmon. This cue is distinctive in our situation in that none of the other fish on the list would bring red to mind. But when the cue red is given at test, its distinctive relation to salmon is defined by the context. In this case, the context is provided by both the instructions to produce words from the list and the category fish. In short, the organizational processing of shared attributes is necessary for the beneficial effects of distinctive processing to emerge. Herein lies the function of organization.

Organizational processing provides information that subsequently allows reinstatement of the episode of which the target event was a member. Theoretically, the organizational information is qualitatively different from distinctive information, and it follows that organizational information is not directly useful for item retrieval. That is, organizational encoding contributes to item memory through the necessary but indirect route of establishing the episodic context for the more specific distinctive information in retrieval. For this reason, organizational processing alone never produces the level of performance that accompanies the combination of organizational and distinctive processing.

What we are advocating is, at least in part, a different view of the function of organization. This view places litthe emphasis on the "grouping" process of encoding and storage that traditionally is the basis of explanation of organizational processing. In fact, as our research and other work we have reviewed indicates, organizational processing is not particularly good for item memory. Organization is not the processing of items and cannot directly facilitate item memory. Rather, organization is the processing of relationships that constitute potential cues specifying a particular episode.

There is, however, another role for organization, and it is one that enables an important function of memory. As our data have shown, organizational processing of familiar events is much more consensual than is distinctive processing. Note that organizational processing could be quite idiosyncratic, as with subjective organization, but with familiar events this is unlikely. In the course of social/ linguistic development, members of a culture come to share perceptions and descriptions of relationships among events. I then am confident of communicating with your memory by offering organizational cues such as, "Who was at the party Saturday night?" The consensuality of organizational processing is essential to the social function of memory, and as such, recommends attention to differences between self-cued and other-cued performance in research. There is no reason to believe that the basic principles of memory differ as a function of cue source, but it is clear that the effects of a particular cue type depend on who provides the cue. Your distinctive cue may be of little use to my memory, but your organizational cue may be quite helpful. The same point has been made in an important study of cue function in communication by Harris, Upfold, and Begg (1982). The social function of memory constrains the effectiveness of particular cues in ways that are different from self-generated retrieval situations.

\section{Constraints on Retrieval}

Intentional retrieval rarely involves a single cue, but rather occurs in the presence of multiple cues. Explicit acknowledgment of multiple cues facilitates our conceptualization of retrieval. For example, Humphreys, Wiles, 
and Bain (1993) have suggested a model of retrieval as the intersection of multiple cues. Rubin and Wallace (1989) provided an elegant demonstration of essentially this point by showing that the combined effect of rhyme and meaning cues produced recall far in excess of performance predicted by the independent effects of the cues.

Distinctive and organizational cues offer a general description of powerful, nonredundant constraints on episodic retrieval. Organizational processing defines an episode in that an episode comprises events sharing a relationship. Specification of the episode is necessary in any request for memory, a point well taken from Tulving (1983). People will not comply with a question about their memory unless the question contains, however implicitly, an episodic constraint. Asking someone, "What did you see?" will at best elicit a request for more constraining cues. We suggest that organizational processing and subsequent organizational cues carry the episodic constraint in that all of the events of the episode share spatial, temporal, and perhaps semantic attributes. "What did you see at the theater on your last visit to New York?"

Organizational processing by definition is shared by multiple items, and consequently, the addition of distinctive processing is necessary for retrieval of a particular item. The distinctive processing adds nonshared information about a particular item aggregated in the organizational processing, and the combination of organizational and distinctive processing allows unique convergence on a particular item of a particular episode. In this regard, the results of Experiment 3 are impressive in showing that the reinstatement of shared cues allows the benefits of distinctive processing to emerge.

This finding offers a clue as to how the coaction of organization and distinctiveness allows memory to be highly adaptive and powerful. Organizational cues are not particularly good when accompanied only by organizational encoding, but organizational cues are likely to be consensual and can reinstate distinctive processing. Thus it is that you can elicit my particular memory with your general cue.

\section{REFERENCES}

Battig, W. F., \& Montague, W. E. (1969). Category norms for verbal items in 56 categories: A replication and extension of the Connecticut category norms. Journal of Experimental Psychology Monographs, 80, (3, Pt. 2).

CraIK, F. I. M. (1979). Levels of processing: Overview and closing comments. In L. S. Cermak \& F. I. M. Craik (Eds.), Levels of processing in human memory (pp. 447-461). Hillsdale, NJ: Erlbaum.

CraIK, F. I. M., \& JACOBY, L. L. (1979). Elaboration and distinctiveness in episodic memory. In L. Nilsson (Ed.), Perspectives on memory research: Essays in honor of Uppsala University's 500th anniversary (pp. 145-166). Hillsdale, NJ: Erlbaum.
CRAIK, F. I. M., \& LockHART, R. S. (1972). Levels of processing: A framework for memory research. Journal of Verbal Learning \& Verbal Behavior, 11, 671-684.

EINSTEIN, G. O., \& HUNT, R. R. (1980). Levels of processing and organization: Additive effects of individual-item and relational processing. Journal of Experimental Psychology: Human Learning \& Memory, 6, 588-598.

EYSENCK, M. W. (1979). Depth, elaboration, and distinctiveness. In L. S. Cermak \& F. I. M. Craik (Eds.), Levels of processing in human memory (pp. 89-118). Hillsdale, NJ: Erlbaum.

HARRIS, G., Upfold, D., \& BEGG, I. (1982). On the role of the speaker's expectations in interpersonal communication. Journal of Verbal Learning \& Verbal Behavior, 19, 597-607.

HumphrEYs, M. S., Wiles, J., \& Bain, J. D. (1993). Memory retrieval with two cues: Thinking of intersecting sets. In D. E. Meyer \& S. Kornblum (Eds.), Attention and performance XIV: A silver jubilee (pp. 489-507). Hillsdale, NJ: Erlbaum.

HunT, R. R., \& Einstein, G. O. (1981). Relational and item-specific information in memory. Journal of Verbal Learning \& Verbal Behavior, 20, 497-514.

HUNT, R. R., \& MCDANIEL, M. A. (1993). The enigma of organization and distinctiveness. Journal of Memory \& Language, 32. 421-445.

Hunt, R. R., \& SETA, C. E. (1984). Category size effects in recall: The roles of individual item and relational information. Journal of Experimental Psychology: Learning, Memory, \& Cognition, 10, $454-$ 464.

KELLY, R. E. S. (1993). Good cues gone bad: Self-generated distinctive cues lose their effectiveness in the absence of explicit recall instructions. Unpublished master's thesis, University of North Carolina, Greensboro.

Lockhart, R. S., Craik, F. I. M., \& Jacoby, L. L. (1976). Depth of processing, recognition and recall. In J. Brown (Ed.), Recall and recognition (pp. 75-102). New York: Wiley.

MäNTYLÄ, T. (1986). Optimizing cue effectiveness: Recall of 500 and 600 incidentally learned words. Journal of Experimental Psychology: Learning, Memory, \& Cognition, 12, 66-71.

MäNTYLÄ, T., \& Nilsson, L. G. (1983). Are my cues better than your cues? Scandinavian Journal of Psychology, 24, 303-312.

MÄNTYLÄ, T., \& Nilsson, L. G. (1988). Cue distinctiveness and forgetting: Effectiveness of self-generated cues in delayed recall. Journal of Experimental Psychology: Learning, Memory, \& Cognition, 14, 502-509.

Medin, D. L., Goldstone, R. L., \& Genter, D. (1993). Respects for similarity. Psychological Review, 100, 254-278

Moscovitch, M., \& Craik, F. I. M. (1976). Depth of processing, retrieval cues, and uniqueness of encoding as factors in recall. Journal of Verbal Learning \& Verbal Behavior, 15, 447-458.

RuBin, D. C., \& WALLACE, W. T. (1989). Rhyme and reason: Analyses of dual retrieval cues. Journal of Experimental Psychology: Learning, Memory, \& Cognition, 15, 698-709.

STEIN, B. (1977). The effects of cue-target uniqueness on cued recall performance. Memory \& Cognition, 5, 319-322.

Tulving, E. (1983). Elements of episodic memory. Oxford: Oxford University Press, Clarendon Press.

Tulving, E., \& Pearlstone, Z. (1966). Availability versus accessibility of information in memory for words. Journal of Verbal Learning \& Verbal Behavior, 5, 381-391.

WatKINS, O. C., \& WATKINS, M. J. (1975). Buildup of proactive inhibition as a cue-overload effect. Journal of Experimental Psychology: Human Learning \& Memory, 104, 442-452.

(Manuscript received June 9, 1994; revision accepted for publication February 3, 1995.) 